\title{
Contrôle qualité de la production de vins tropicaux malgaches en provenance de la région de Haute Matsiatra, au sud de Madagascar
}

\author{
Tahina Lalaina RANDRIANANTOANDR0 ${ }^{1}$, Tahina ANDRIAMAMISA ${ }^{1}$ \\ ${ }^{1}$ Laboratoire de Chimie de l'Environnement - Faculté des Sciences- Université d'Antananarivo, BP 906- Antananarivo \\ 101 - Madagascar \\ Auteur correspondant : rtla@moov.mg
}

Original submitted in on 24th October 2017. Published online at www.m.elewa.org on $28^{\text {th }}$ February 2018 https://dx.doi.org/10.4314/jab.v122i1.4

\section{RESUME}

Objectif: La filière "vin » est l'un des premiers pôles économiques de la région de Haute Matsiatra (Madagascar), après la riziculture. Tous les acteurs cherchent constamment à promouvoir ce secteur afin d'améliorer la qualité des produits et augmenter par la suite la commercialisation de ces vins au niveau national, voire international. Nos recherches permettent d'établir une bonne traçabilité qualitative des vins tropicaux de Madagascar, à tous les niveaux de chaîne de production (cépage, production et commercialisation).

Méthodologie et résultats: Des paramètres physico-chimiques et bactériologiques sont déterminés pour permettre le contrôle qualité de différents types de vin (rouge, blanc sec, gris, blanc moelleux, rosé et apéritif). L'influence « climat » affecte la préparation du vin. La forte pluviométrie avant le vendage réduit le taux de sucre du raisin. On a souvent recours à la chaptalisation, surtout pour le vin rouge. Pour les vins produits, on suspecte un taux d'acidité volatile très élevé $(2,254$ à 4,067 $\mathrm{g} / \mathrm{L})$ par rapport aux normes internationales. La faible teneur en anhydride sulfureux ( $<150 \mathrm{mg} / \mathrm{L}$ pour les vins rouges, $<200 \mathrm{mg} / \mathrm{L}$ pour les vins blancs) influence la stabilité du vin à la conservation. L'acide tartrique régule l'acidité du vin, mais il peut se précipiter en bitartrate de potassium à basse température (stockage à froid). Les différents types de vins analysés ont une teneur en bitartrate de potassium assez élevé, de 13,14 à 21,58 g/L. Cette teneur est faible dans les vins de qualité. Les analyses bactériologiques montrent qu'aucune altération biologique n'est observée dans les vins échantillonnés.

Conclusion et application des résultats : Les acteurs nationaux suggèrent la promotion effective de la filière de la part de l'État. La création d'un laboratoire agréé et la mise en place d'une législation spécifique sont donc attendu pour recadrer le secteur.

Mots-clés : cépage, chaptalisation, anhydride sulfureux, contrôle qualité, vin malgache, région de Haute Matsiatra, Fianarantsoa - Madagascar 


\section{Quality control of tropical wine production from the region of Haute- Matsiatra, in south of Madagascar ABSTRACT}

Objectives: Wine production is an economic focus in Haute Matsiatra district (Madagascar). Local actors were investigated to promote the wine sector and to improve the quality of products for national commercialization or on international level. This research allows establishment of a traceability study of the wine quality product, in the chain of the production level (grape production and commercialization).

Methodology and results: physico-chemical and bacteriological parameters are used to determine the quality control of different types of wines (red wine, dry white wine, gray wine, sweet white wine, rose wine and aperitif wine). Climate affects the preparation of wine. The high rainfall reduces the sugar level. Therefore, they often resort to chaptalization, especially for red wine. In the product, a very high of volatile acidity $(2,254$ to $4,067 \mathrm{~g} /$ $\mathrm{L}$ ) is suspected, compared to international standards. The low sulfur dioxide content ( $<150 \mathrm{mg} / \mathrm{L}$ for red wines, $<200 \mathrm{mg} / \mathrm{L}$ for white wines) influence the stability of wine conservation. The tartaric acid regulates the acidity of wine, but it may precipitate as potassium bitartrate in a low temperature condition (cold storage). A different type of wine analyzed have a high level of potassium bitartrate, 13,14 to $21,58 \mathrm{~g} / \mathrm{L}$. It was low in the best wine. Bacteriological analyzes indicated that there was no biological alteration in the wines sampled.

Conclusion and application of results: National actors suggest the effective promotion of the sector from the authority. Creating an approved laboratory and the establishment of specific legislation are expected to refocus the wine sector.

Keywords: grape variety, chaptalization, sulfur dioxide, quality control, malagasy wine, region of Haute Matsiatra, Fianarantsoa - Madagascar

\section{INTRODUCTION}

Le vin est défini comme étant « le résultat exclusif de la fermentation du raisin frais » par la loi Griffe du 14 Août 1889, celle du 3 Septembre 1907 ajoutant au raisin «le jus de raisin frais ». La loi française est reprise par le règlement du 28 Avril 1970 en précisant que le vin est le produit obtenu exclusivement par la fermentation alcoolique, totale ou partielle, de raisins frais, foulés ou non, ou de moûts de raisins (Garrier, 1998). Les pays européens sont les plus grands producteurs de vins dans le monde avec 48,9 MhL pour l'Italie, 47,4 MhL pour la France, 36,6 MhL pour l'Espagne. Quant aux autres continents comme le continent américain, on a 22,1 MhL pour les États-Unis, 13,4 MhL pour l'Argentine et 12,9 MhL pour la Chili. Pour l'Océanie, il y a l'Australie avec une production annuelle de 12 MhL. L'Afrique du Sud représente le continent africain avec 11,3 MhL de vin par an. Enfin, le continent asiatique dont la Chine avec une production annuelle de 11,2 MhL de vins (OIV, 2015). Madagascar aussi est un pays producteur de vin. Les terroirs malgaches se trouvent principalement dans la province de Fianarantsoa, région de Haute Matsiatra. Au 19ème siècle, des Français ont implanté les premiers vignobles dans cette région, mais actuellement, on y trouve aussi des Chinois qui ont secouru cette production après plusieurs phases de difficulté, économique et financière. Au début, cette viticulture a été introduite à Madagascar pour produire les vins de messe. $\mathrm{A}$ nos jours, les vins commencent à prendre place dans le quotidien des Malgaches. On a apprécié les différentes variétés existant sur le marché local: rouge, blanc, gris, avec des textures différentes dont le moelleux et le mousseux, sans oublier le vin apéritif qui commence à entrer dans nos mœurs. Plusieurs recherches ont été déjà effectuées pour l'étude de vin à Madagascar, mais aucune donnée scientifique n'a pas encore mis au point la qualité de vin local. En 1999, Rajaona a effectué l'étude physico-chimique des vins rouges commerciales à Madagascar. La même année, une autre étude a été effectuée par Ndrasana (1999), celle-ci concerne la recherche des falsifications des vins blancs commercialisés sur le marché. Enfin, des extraits des vins en provenance des quatre localités de 
Madagascar ont été analysés par résonance magnétique pour identifier et quantifier des composés osidiques dans les produits (Razafindraibe, 2010). Actuellement, les vins malgaches font face à un problème sur le marché mondial en raison de leur qualité, selon des connaisseurs de vin. De ce fait, les vins sont vendus seulement sur le marché local. Quelques sociétés ont aujourd'hui commencé à conquérir le défi pour mettre au berceau les vins malgaches, et mettre un label vis-à-vis des produits importés d'Afrique du sud, de France et de Suisse. Selon Krug Johan Joseph (1800-1866), célèbre producteur de champagne, " le bon vin naît d'un bon raisin, de bons fûts, d'une bonne cave et d'un honnête homme pour coordonner l'ensemble ». Cette citation nous indique quelques facteurs influençant la qualité des

\section{MATERIELS ET METHODES}

Présentation du site d'étude : La société de production de vin échantillonné est une société vitivinicole employant 625 actionnaires qui sont tous des paysans viticulteurs, 85 personnels permanents avec 1500 familles viticulteurs. Elle a un capital de 100.000.000Ar (environ 26.667 €). La produits de vin, dès la viticulture à la production. Notre investigation permet donc d'étudier l'interdépendance de la qualité de vin avec des conditions climatiques de la région, de suivre les procédés de fabrications ainsi que de contrôler la qualité des vins par les analyses physico-chimiques et bactériologiques de différentes variétés de produits avant la mise en bouteilles. L'étude a été réalisée en collaboration avec une société locale, dénommée "Lazan'i Betsileo », premier producteur de vin de la région Haute Matsiatra, voire Madagascar, afin d'améliorer la qualité de sa production pour conquérir le marché mondial. Il est à noter que la société a obtenu en 2003 le Golden Europe Award for Quality et le New Millénium Award à Paris.

société vend ses produits en détail et en gros selon les clients. La capacité de production de la société reste limitée. II ne produit que 800 tonnes de raisins par an pour fabriquer 500000 litres de vin. 


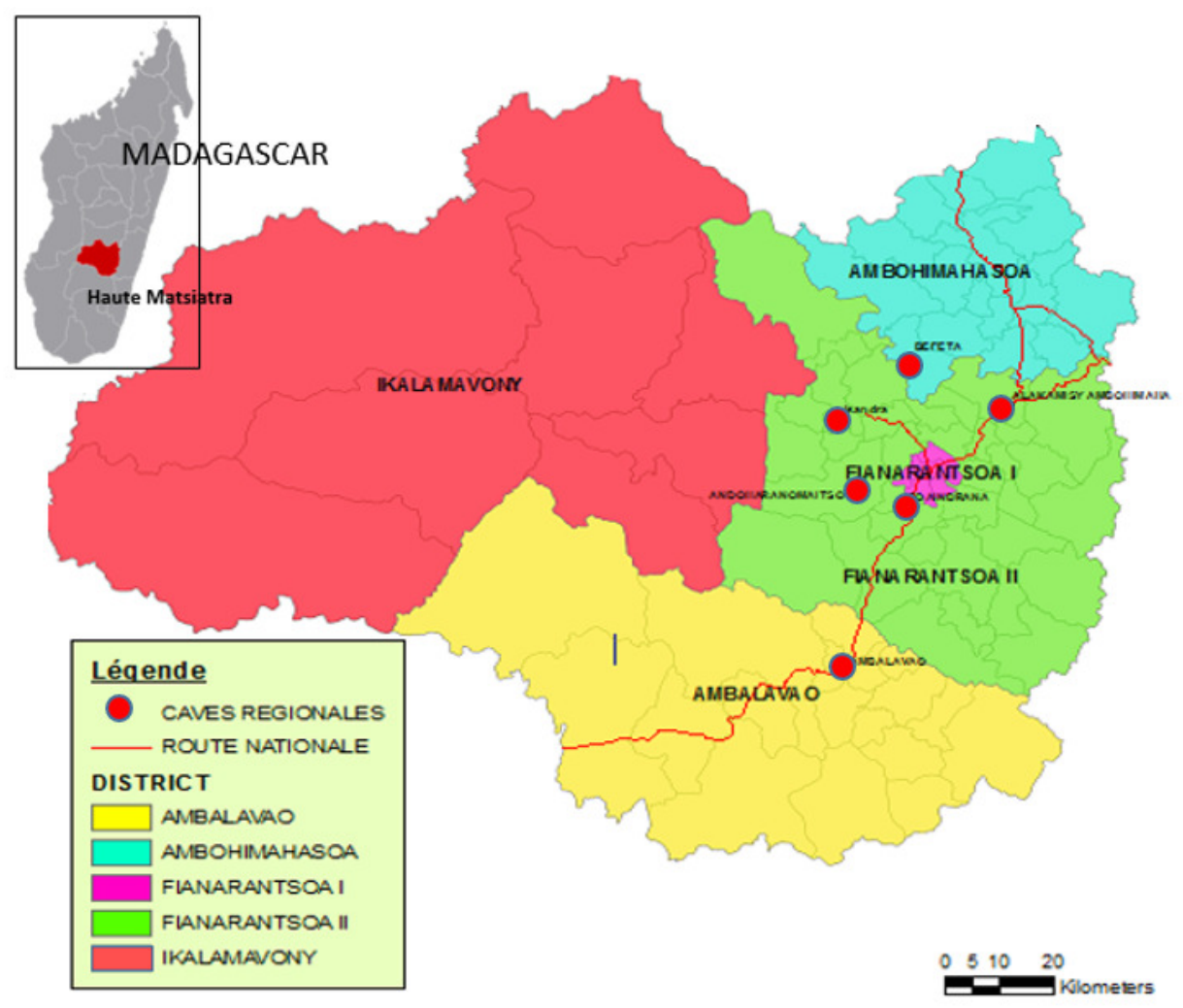

Carte 1: Localisation des viticultures dans la région de Haute Matsiatra

- Condition climatique : La région Haute Matsiatra est une région possédant une importante potentialité, tant en ressources humaines que sur le plan économique en général. Sa situation géographique, ne la défavorisant pas, au contraire, l'incite à se développer. En effet, le terroir perché à plus de $1200 \mathrm{~m}$ d'altitude, bénéficiant d'un très bon ensoleillement et d'une hygrométrie idéale favorise la filière vin de la région. Ce secteur intéresse même les investisseurs chinois qui y louent des parcelles de vignobles. Haute Matsiatra est dominée par une vaste colline où les vignobles se localisent sur les coteaux. Des pentes et des vallées assez larges se succèdent tout le long du relief. Le climat de la Région Haute Matsiatra est du type tropical des Hautes Terres, et se subdivise en trois saisons : saison froide et sèche, accompagnée des crachins matinaux de temps en temps (Avril-Août) ; saison chaude et sèche (Septembre-Octobre) ; saison chaude et pluvieuse (Décembre-Mars). Janvier et Février sont les mois les plus chauds, avec une moyenne de $23,9^{\circ} \mathrm{C}$, tandis que ceux les plus froids sont les mois de Juillet et Août avec une moyenne de $16,7^{\circ} \mathrm{C}$. La température moyenne annuelle est de $20,9^{\circ} \mathrm{C}$. En termes de pluviométrie, Haute Matsiatra reçoit $1386 \mathrm{~mm}$ de précipitation par an, dont $88 \%$ tombent du mois d'Octobre à Mars, et le reste, d'Avril à Septembre. Janvier est le mois le plus arrosé, avec une précipitation moyenne de $326,5 \mathrm{~mm}$, alors que le mois d'Août ne reçoit que 11,3 $\mathrm{mm}$ de pluie.

- Viticulture : La viticulture est un domaine que les paysans de la région de Haute Matsiatra pratiquent comme culture de rente, à part le riz. On la voit presque dans chaque district. La culture de la vigne a besoin de conditions climatiques et de sols spécifiques pour qu'on ait une bonne production. Malgré la pauvreté en industrie de la région, la viticulture y est propice. La Société cultive ses propres vignes sur une surface de 300 ha dans son domaine central à Soaindrana situé à $13 \mathrm{~km}$ de la ville de Fianarantsoa. Toutefois, elle bénéficie aussi de caves régionales localisées à Befeta, Isandra, Alakamisy Ambohimaha, Andoharanomaitso, Ambalavao (Carte 1). Les cépages des vignobles sont tous des hybrides du type Vitis vinifera tels que :

- le Petit Bouchet : donne un vin très coloré, plat et peu alcoolique,

- le 26205 ou Joannès- Seyve ou Chambourcin,

- le 23657 ou Seyve-Villard ou Varoussset, 
- le 18315 ou Seyve-Villard ou Villard noir : donne un vin alcoolique, coloré, astringent avec un goût herbacé assez prononcé.

- le Couderc 13 : donne au vin une odeur rappelant la cannelle, légèrement pommadé.

Les paramètres analysés :

Sucre et alcools : L'évaluation des teneurs en sucres des moûts concentrés et des vins ainsi que la mesure de titre alcoométrique probable du vin (TAVP) ont été effectuées par réfractométrie. La teneur en sucre réducteur a été déterminée par titrimétrie avec de l'iodure de potassium en présence de sulfate de cuivre. Le degré alcoométrique est déterminé par densimétrie. La teneur en éthanol a été déterminée par ébulliométrie.

Acidité : Les $\mathrm{pH}$ de chaque vin ont été déterminés car ceux-ci influent grandement sur les propriétés du vin, par exemple sur sa couleur, son oxydation, ainsi que sur sa stabilité biologique et chimique.

L'acidité totale du vin est l'un des constituants essentiels de ses propriétés organoleptiques aussi bien que de sa tenue et sa conservation. II est donc important de suivre ce paramètre périodiquement tout au long de la vinification, surtout pendant la fermentation alcoolique et malolactique, mais aussi jusqu'à la mise en bouteilles. Le dosage de l'acidité total est effectué avec de la soude caustique. Elle est exprimée en $\mathrm{g} / \mathrm{L}$ d'acide sulfurique $\left(\mathrm{H}_{2} \mathrm{SO}_{4}\right)$ ou d'acide tartrique (Schopfer, 1998). Sa valeur moyenne doit être de $4 \mathrm{~g} / \mathrm{L}$ de $\mathrm{H}_{2} \mathrm{SO}_{4}$ soit $6 \mathrm{~g} / \mathrm{L}$ d'acide tartrique (Navarre 1998, OIV 2006). En plus, la teneur en acidité volatile est obtenue par titrage des acides volatiles séparés du vin par entraînement à la vapeur d'eau et rectification des vapeurs. L'acidité fixe est donc déterminée par la différence entre l'acidité totale et

\section{RESULTATS ET DISCUSSION}

Le titre alcoométrique volumique probable (TAVP): Les sucres sont parmi les constituants du raisin. Ils sont présents dans les moûts à des taux différents. La richesse en sucres des moûts permet d'estimer le degré probable de la récolte. Après fermentation complète, la concentration en sucre représente la teneur en sucres résiduels du vin. Les teneurs en sucre mesurées dans les moûts sont $176 \mathrm{~g} / \mathrm{L}$ pour le raisin rouge et $178,3 \mathrm{~g} / \mathrm{L}$ pour le raisin blanc, alors que le moût de raisin contient en moyenne entre 150 et $250 \mathrm{~g}$ de sucre par litre (Vin Social Club 2014, Escudier 2016). Les moûts de raisins blancs contiennent plus de sucres que le moût de raisins rouges. Cette valeur est due à la bonne maturation de la vendange. Le taux de sucre des moûts est suffisant pour obtenir le degré alcoolique nécessaire pour le futur vin. l'acidité volatile. Enfin, le sel de bitartrate de potassium est déterminé par précipitation.

Anhydride sulfureux $\left(\mathrm{SO}_{2}\right)$ : L'anhydride sulfureux est le seul antiseptique autorisé par la loi qui protège le vin à la fois contre l'oxydation et contre les maladies bactériennes. II est donc très important de le doser périodiquement avec exactitude pour suivre son évolution tout au long de l'élevage d'un vin, de la vendange à la mise en bouteilles. L'acide sulfureux est déterminé par iodométrie. Les analyses sont effectuées avant et après sulfitage.

Extrait sec: L'extrait sec est déterminé par pesée du résidu après l'évaporation du vin.

Paramètres microbiologiques: L'analyse microbiologique des vins a pour but de suivre les fermentations alcoolique et/ou malolactique et de déceler les risques d'altérations microbiennes. Ce qui permet ensuite de détecter toute anomalie, non seulement dans le produit fini, mais aussi pendant les différentes phases de sa fabrication (OIV, 2015). Les principaux germes analysés sont le Mycoderma vini, le Mycoderma aceti, le ferment de la tourne, le ferment de l'amertume, les ferments de la graisse. Afin de se conformer à la norme nationale, les analyses microbiologiques ont été réalisées au sein du laboratoire ACSQDA (Agence de Contrôle de la Sécurité Sanitaire et de la Qualité des Denrées Alimentaires) du Ministère de la Santé Publique de Madagascar.

Échantillonnage : Les analyses sont effectuées à partir des moûts de vin et des différents types de vin produit: moût de raisin rouge (MRR), moût de raisin blanc (MRB), vin rouge (VRO), vin blanc sec (VBS), vin gris (VGR), vin rosé (VRE), vin blanc moelleux (VBM), vin apéritif (VAP).

Une chaptalisation n'est donc pas normalement nécessaire lors du processus de vinification. Les TAVP des moûts concentrés rouge et blanc déterminés sont respectivement $10,5 \%$ et $10,6 \%$.

Les $\mathrm{pH}$ et acidités des vins : Les $\mathrm{pH}$ de vins analysés varient de 3,7 à 5 (figure 1). La stabilité chimique et biologique du vin dépend largement de ces pH. Des pH plus acide sont connus par leur effet favorable sur la stabilité du vin et sont favorable à l'inhibition de la prolifération bactérienne, à l'équilibre de la fermentation de sucres. Un vin au $\mathrm{pH}$ faible est également plus attrayant visuellement (Gerbaud 1996 , Akin 2008). En effet, les vins rouges et blancs conservent une couleur plus intense. Les vins rouges ont ainsi une couleur (violet intense) et une odeur plus agréables, et les vins blancs 
ne brunissent pas aussi aisément. Par contre, lorsque le $\mathrm{pH}$ du vin est élevé, les bactéries y prolifèrent rapidement (Cole-Parmer, 2017), et le problème indésirable de la fermentation bactérienne devient plus préoccupant. Les maladies se déclarent plus facilement à un $\mathrm{pH}$ supérieur à 4 . Cette condition diminue la stabilité biologique et chimique du vin. A cet effet, les bactéries (lactiques ou acétiques) peuvent se développer dans le vin en provoquant des altérations plus ou moins graves. Les vins au $\mathrm{pH}$ plus élevé nécessitent toujours plus d'attention et de soins.

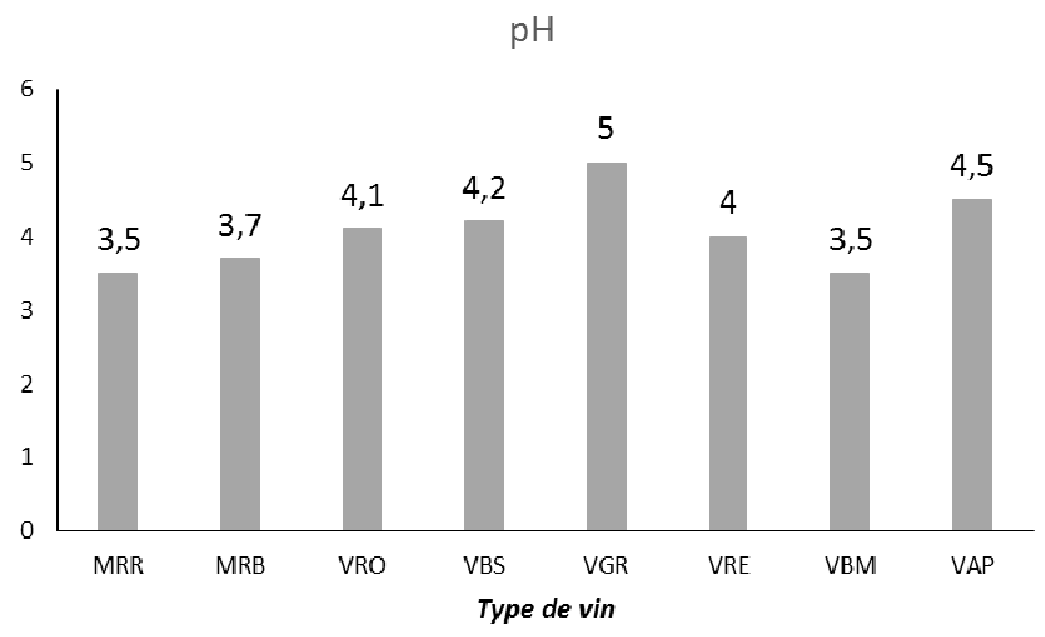

Figure 1: Valeurs des pH de différents types de vins

L'acidité totale des produits analysés varie de 4,5 à 5,7 $\mathrm{g} / \mathrm{L}$ d'acide tartrique. La norme internationale exige une teneur de 3,5 g/L d'acide tartrique au minimum (UE 2009, Bagard 2010, IGP 2011) . L'acidité totale est liée à l'ensemble des acides présent dans le vin. Elle traduit les caractéristiques gustatives du vin (Navarre, 1998). En effet, une faible acidité correspond à l'objectif général des grands vins tandis qu'une acidité élevée facilite la conservation en cas d'hygiène précaire. Elle masque un peu le manque de charpente et donne de la fraîcheur aux vins blancs et rosés. Cette acidité joue un rôle important sur les qualités organoleptiques du vin mais c'est aussi un paramètre de suivi de la maturation du raisin (dosage du moût) et de la fermentation malolactique. Elle joue également un rôle dans la conservation du vin.

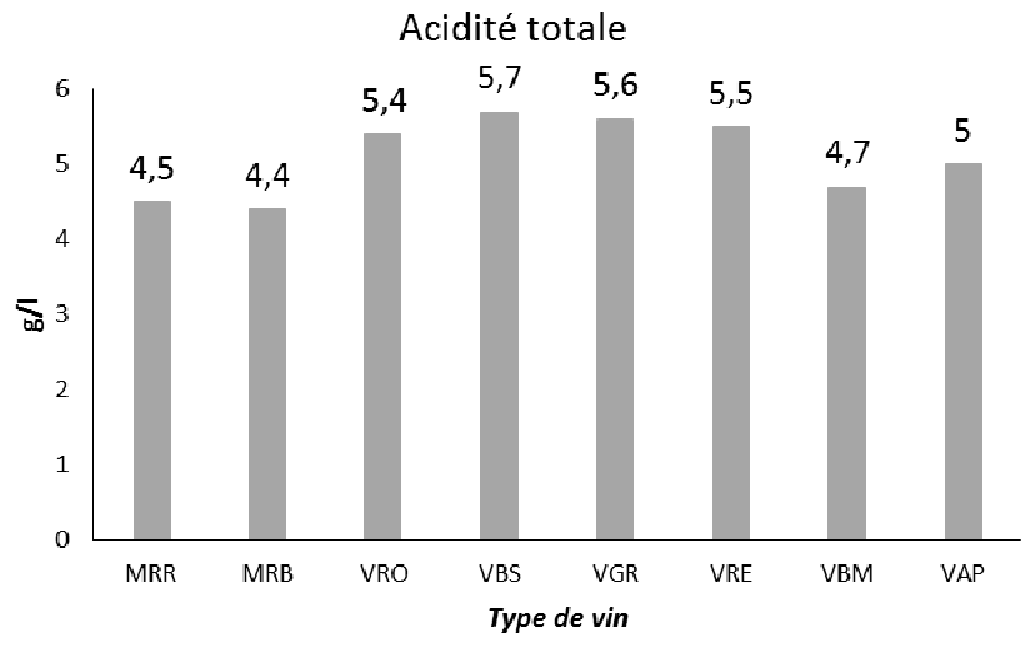


Figure 2: Valeurs de l'acidité totale de différents types de vins

L'acidité volatile est constituée par la partie des acides gras appartenant à la série acétique, qui se trouvent dans les vins à l'état libre, et à l'état salifié. Elle est formée essentiellement par l'acide acétique, accompagné de petites quantités d'acides propionique et butyrique. Le $\mathrm{CO}_{2}$ et le $\mathrm{SO}_{2}$ ne font pas partie de l'acidité volatile. L'acidité volatile est un paramètre important de la qualité des vins (Delanoë D. et al., 2007). Elle donne du bouquet au vin. La valeur de l'acidité volatile des six échantillons de vins analysés varie de 2,254 à 4,067 g/L d'acide sulfurique, alors que les valeurs proposées par la règlementation internationale sont de $0,88 \mathrm{~g} / \mathrm{L}$ au maximum pour les vins blancs et $0,98 \mathrm{~g} / \mathrm{L}$ au maximum pour les vins rouges. Ces acides se forment naturellement en très faible quantité pendant les fermentations alcoolique et malolactique. Elles peuvent aussi se former accidentellement suite au développement bactérien. Mais lorsque la dose est trop élevée, comme dans notre cas, le vin devient trouble et piqué. La détermination de l'acidité volatile d'un vin permet aussi de connaître l'état sanitaire des vins.

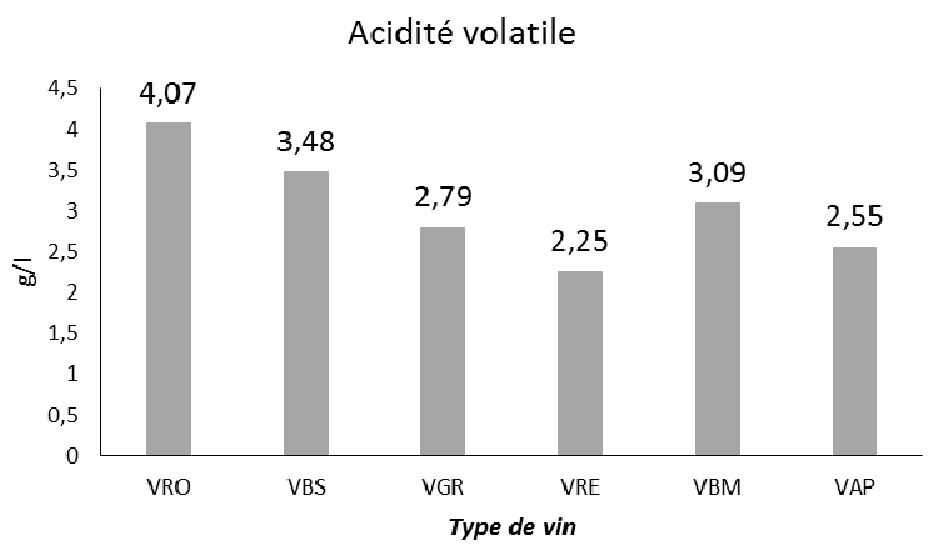

Figure 3: Valeurs de l'acidité volatile de différents types de vins

Selon le type de vin, la valeur de l'acidité fixe est comprise entre 0,735 et $1,617 \mathrm{~g} / \mathrm{L}$ d'acide sulfurique. II

n'existe aucune norme limitant la quantité d'acidité fixe contenue dans le vin.

\section{Acidité fixe}

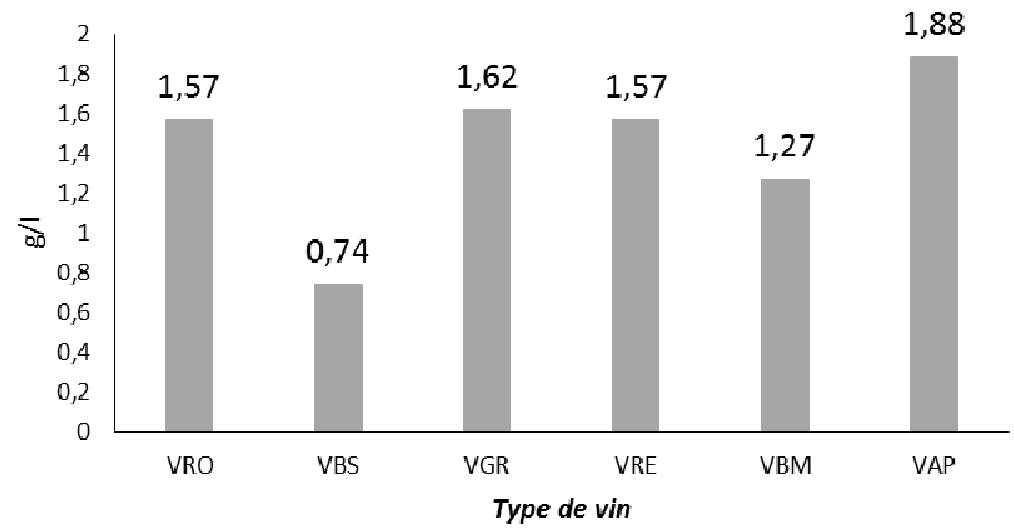

Figure 4: Valeurs de l'acidité fixe de différents types de vins 
Teneur en anhydride sulfureux : $\mathrm{Le} \mathrm{SO}_{2}$ libre est la fraction du $\mathrm{SO}_{2}$ incorporé dans le moût ou dans le vin. II est le seul antiseptique ou antioxydant autorisé en vinification. Le manque d'anhydride sulfureux peut provoquer l'apparition d'un caractère éventé du vin à la dégustation (Delanoë D. et al., 2007). Dans le vin, une grande partie du $\mathrm{SO}_{2}$ se combine avec les sucres, les matières colorantes, les vitamines, les acides et l'acétaldéhyde. Au cours de la fermentation, les vins rouges s'enrichissent en divers éléments libérés par les matières solides dont les polyphénols qui sont capables de fixer l'oxygène dissous dans le vin et donc d'éviter l'oxydation. Les vins rosés et blancs qui fermentent sans la peau, pépins et rafles sont donc plus oxydatifs. Les vins contenant des sucres qui combinent le $\mathrm{SO}_{2}$, nécessitent des quantités importantes de $\mathrm{SO}_{2}$ total pour avoir un peu de $\mathrm{SO}_{2}$ libre (ITV France 2002, Delanoë D. et al. 2007). C'est pour cela que les vins rouges contiennent moins de $\mathrm{SO}_{2}$ que les autres types de vins. Pendant l'élevage de vin, des valeurs de $\mathrm{SO}_{2}$ libre de 25 $\mathrm{mg} / \mathrm{L}$ pour le vin rouge et de $30 \mathrm{mg} / \mathrm{L}$ pour le vin blanc sont à recommander. De plus, il est admis en œnologie qu'une dose de $15 \mathrm{mg} / \mathrm{L}$ de $\mathrm{SO}_{2}$ libre est efficace pour protéger le vin. Cependant, le dioxyde de soufre demeure un produit dangereux et toxique pour les utilisateurs qu'il convient de manipuler avec précaution. Les teneurs en $\mathrm{SO}_{2}$ libre dans les différents types de vins échantillonnés après sulfitage varient de 30 à $35 \mathrm{mg} / \mathrm{l}$ selon le type de vin (figure 5), alors que la norme malgache ainsi que la norme internationale exige une teneur de $40 \mathrm{mg} / \mathrm{l}$ au maximum à la consommation. Quant au teneur de l'anhydride sulfureux total, il varie de 105 à $171 \mathrm{mg} / \mathrm{l}$ si la norme préconisée est de $250 \mathrm{mg} / \mathrm{l}$. La teneur en $\mathrm{SO}_{2}$ totale des différents types de vins analysés est comprise entre 105 et $171 \mathrm{mg} / \mathrm{L}$. L'anhydride sulfureux total de ces vins est normal puisque c'est inférieur à la valeur limite fixée par la loi n95-022 adoptée par l'Assemblée Nationale Malagasy qui est de $250 \mathrm{mg} / \mathrm{L}$. Par rapport à la norme internationale, l'anhydride sulfureux total de vin rouge est supérieur à $150 \mathrm{mg} / \mathrm{L}$, la valeur limite tandis que le $\mathrm{SO}_{2}$ total des vins blancs et rosés analysés est conforme à la norme qui est de $200 \mathrm{mg} / \mathrm{L}$.

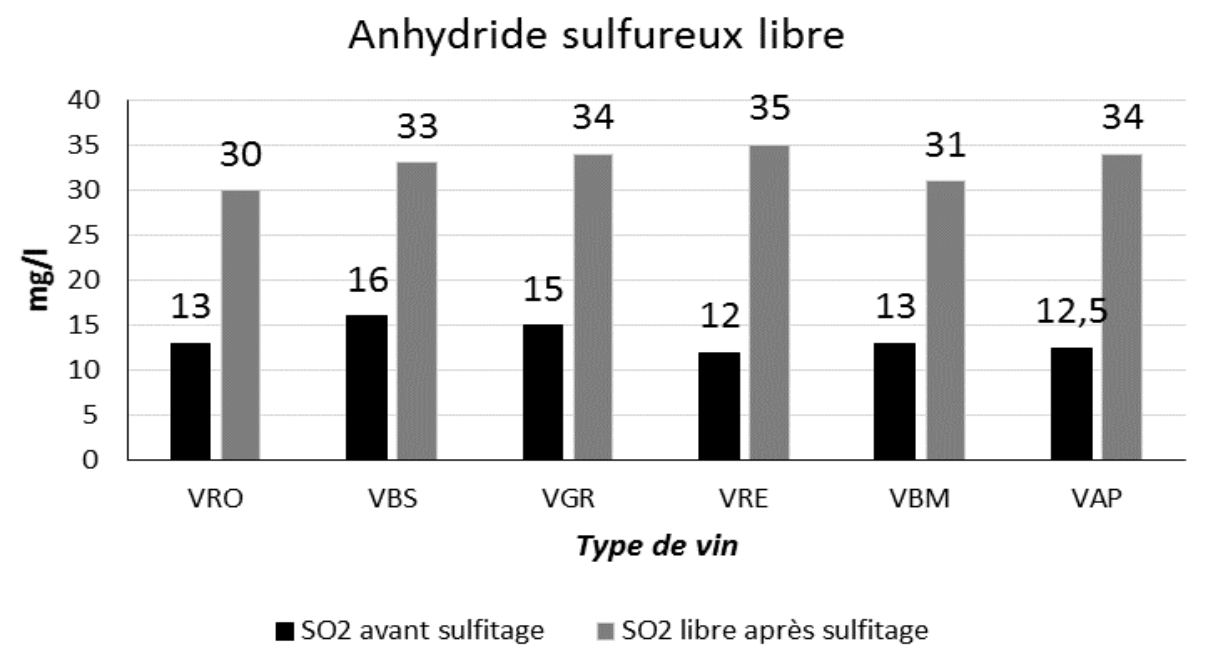

Figure 5 : Teneur de l'anhydride sulfureux libre dans les vins 


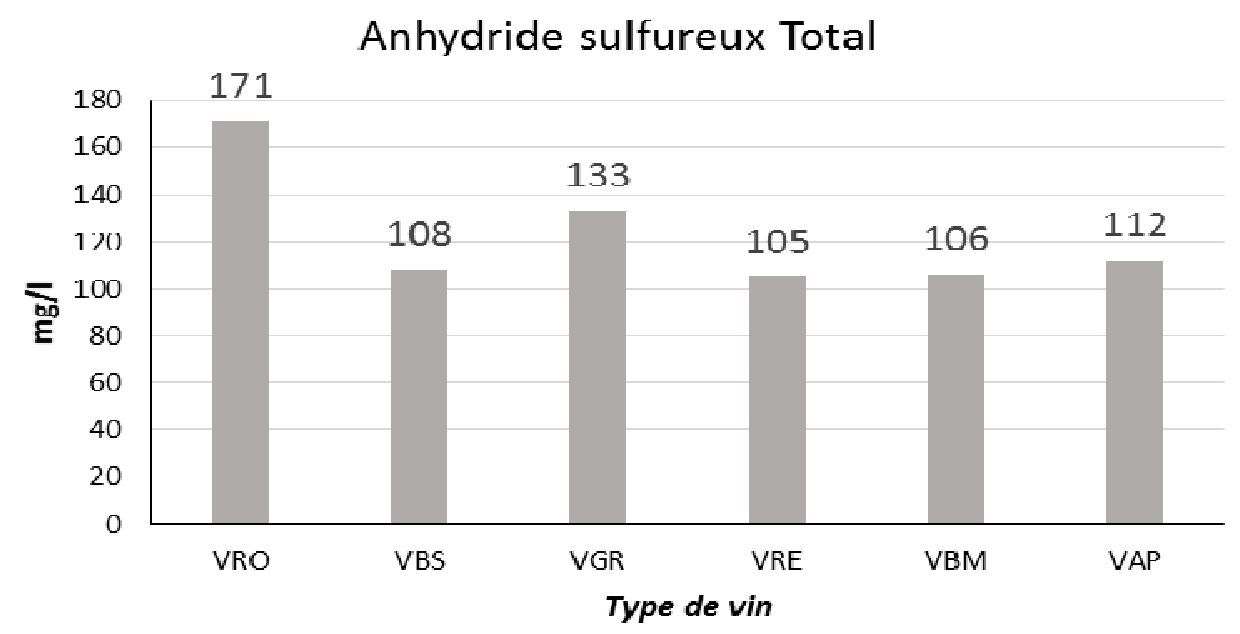

Figure 6 : Teneur de l'anhydride sulfureux total dans les vins

Degré alcoolique des vins : L'alcool dans un vin est le résultat de la transformation, totale ou partielle, du sucre contenu dans le raisin. Le degré alcoolique du vin peut varier selon la méthode d'élaboration et les pays d'origine. Les degrés alcooliques des vins échantillonnés en provenance de la région Haute Matsiatra varient de 11 et $14,7 \%$. Pour les autres types de vin, il est au voisinage de $11 \%$. D'après la loi nº5-022, l'Assemblée Nationale Malagasy fixe le taux d'alcool du vin à $8 \%$ vol au minimum, alors que la norme européenne fixe la valeur limite du titre alcoométrique volumique acquis à $9 \%$ minimum. Le vin rouge a un degré alcoolique de $11 \%$, présentant un teneur en sucre de $13,88 \mathrm{~g} / \mathrm{l}$ qui donne un goût particulièrement caractéristique. Pour le vin blanc sec, le degré d'alcool est de $11,8 \%$, avec une teneur en sucre de $3 \mathrm{~g} / \mathrm{l}$. Un vin blanc sec type bordeaux, par exemple, possède une teneur en sucre inférieur à $4 \mathrm{~g} / \mathrm{l}$. Par contre, les vins gris et rosé affichent des degrés alcooliques 11,5 et $11,2 \%$, avec des teneurs en sucre de 4,11 et $5,18 \mathrm{~g} / \mathrm{l}$. Pour les vins blancs moelleux et apéritifs, les degrés alcooliques sont élevés (figure 7) et ils possèdent une concentration en sucre résiduel élevée : $90,43 \mathrm{~g} / \mathrm{l}$ pour le vin blanc moelleux et $111 \mathrm{~g} / \mathrm{l}$ pour le vin apéritif. En général, les vins moelleux doivent avoir une teneur en sucre entre 40 à $60 \mathrm{~g} / \mathrm{l}$. Alors, au-delà de cette concentration, les vins sont dites liquoreux à cause de goût amer et sucré. En réalité, le degré alcoolique de vin varie d'un pays à un autre. Ceci dépend de la condition climatique de la région, l'élevage et les méthodes d'élaboration du vin.

\section{TAV acquis}

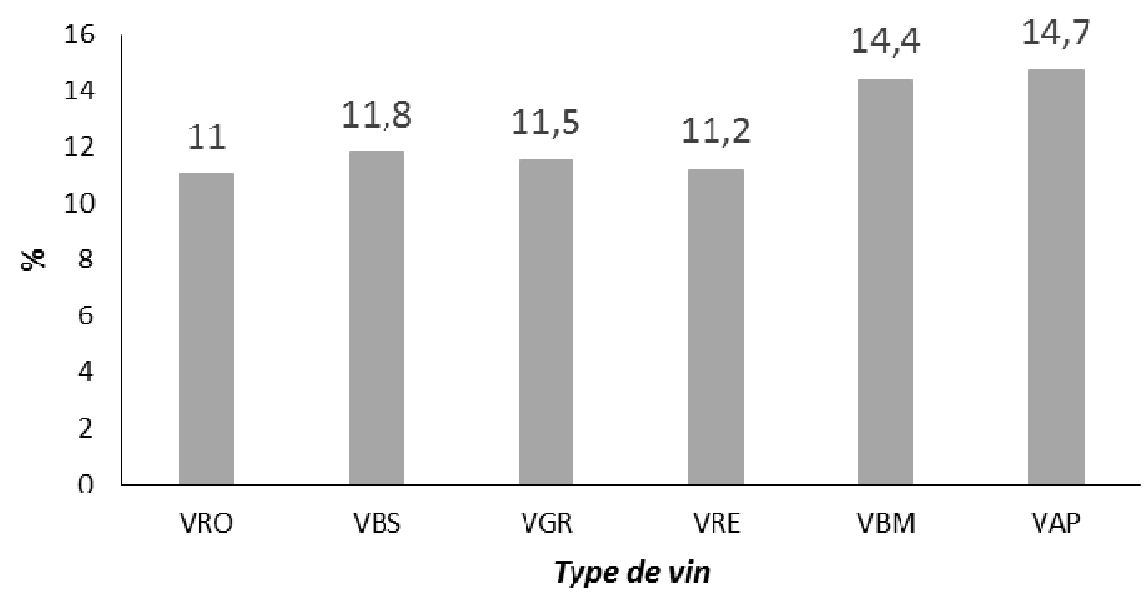


Figure 7 : Titre alcoométrique volumique des vins

Teneur en bitartrate de potassium : La présence des acides organiques donne la qualité de vin mais aussi leur défaut. L'acide tartrique régule l'acidité de vin, mais peut se précipiter sous forme de bitartrate de potassium sous l'action de la température (conservation à froid). Le pH d'un vin dépend beaucoup de la teneur en acide tartrique. La concentration de cet acide diminue par précipitation de bitartrate de potassium au cours de la fermentation puis sous l'action du froid, et ensuite plus lentement par précipitation de tartrate neutre de calcium. Les différents types de vins analysés ont une teneur en bitartrate de potassium variant de 13,14 à $21,58 \mathrm{~g} / \mathrm{L}$ (figure 8 ). Le bitartrate de potassium ne comporte aucune réglementation particulière. Cet intrant est un additif alimentaire, que le Codex Alimentarius classe en stabilisant et en séquestrant. Lorsque la teneur en bitartrates est au-dessus du point de saturation, on pourrait avoir une précipitation tartrique à basse température. Certains consommateurs n'apprécient pas les dépôts au fond des bouteilles. La réduction de la teneur en bitartrate de potassium dans le vin permet d'optimiser le traitement du vin à froid (Tisseau 1992, Gerbaud 1996). Les vins de qualité sont en général pauvres en acide tartrique.

\section{Bitartrate de potassium}

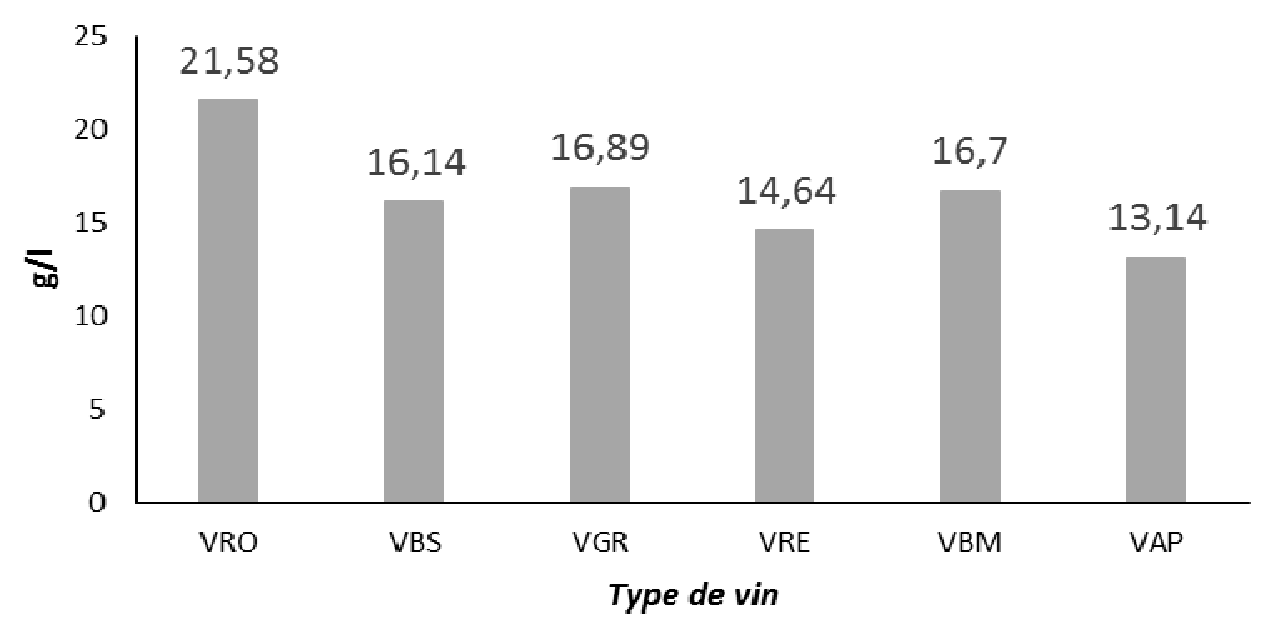

Figure 8 : Teneur en bitartrate de potassium dans les vins

L'extrait sec du vin: L'extrait sec représente les matières constitutives d'un vin. Son analyse permet d'apprécier l'authenticité du vin. Les résultats d'analyse de l'extrait sec des différents types de vins montrent une teneur allant de 11,63 (pour le vin blanc) à $111 \mathrm{~g} / \mathrm{L}$ (pour le vin apéritif). On constate que la valeur de l'extrait sec du vin rouge est plus élevée que celle du vin blanc. Selon la norme fixée par les règlements européens, l'extrait sec d'un vin doit être en moyenne compris entre 17 et $30 \mathrm{~g} / \mathrm{L}$. 


\section{Extrait sec}

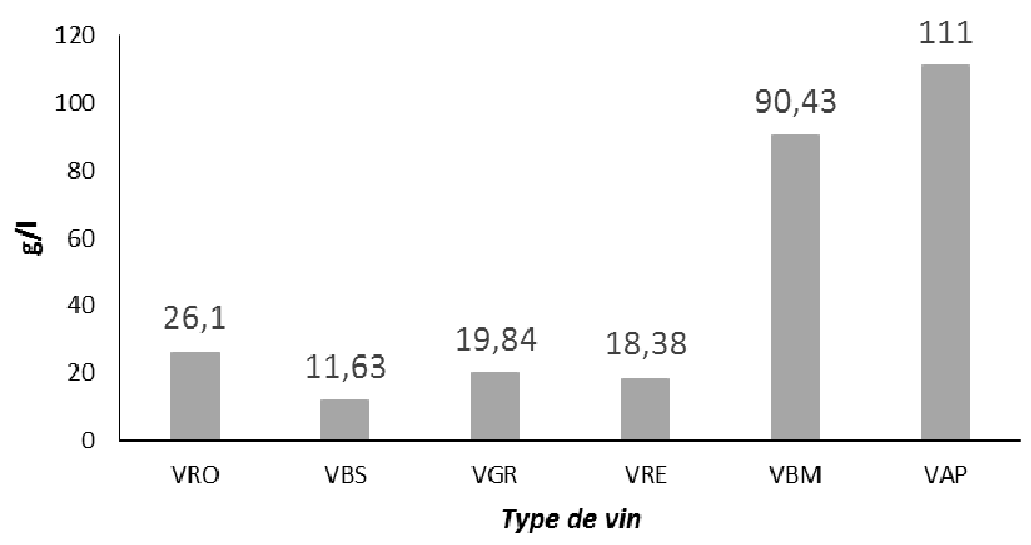

Figure 9 : Valeur de l'extrait sec de différents types de vins

Contrôle microbiologique : Réalisé de façon préventive, les analyses microbiologiques ont permis d'examiner les différents paramètres d'altération caractéristiques des vins. Lorsque ces germes d'altération atteignent le niveau de population préjudiciable, ils pourraient contaminer et entrainer la dégradation physique, chimique et organoleptique des vins. Les analyses des six échantillons de vins (rouge, blanc sec, gris, rosé, blanc moelleux, apéritif) ont révélé l'absence totale de populations bactériennes responsables de la dégradation biologique des vins. Cela signifie que les vins échantillonnés ne présentent aucun risque de contamination et sont stables. Parmi les germes analysés, on a identifié la présence de Mycoderma vini qui pourrait transformer l'alcool du vin en eau et en acide carbonique pour former à la surface une pellicule blanche appelée « fleur ». On identifie aussi le Mycoderma aceti qui pourrait transformer l'alcool du vin en acide acétique et sera responsable de la piqûre du vin ainsi que de sa transformation en vinaigre (Harry 1996, Carette 2005, Hudelson 2011). Enfin, le ferment de la tourne, le ferment de l'amertume et le ferment de la graisse sont des agents responsables des maladies lactiques des vins. Ces maladies sont dues à la présence de ces bactéries qui dégradent, pour subsister dans le vin, différents composés du vin autres que l'acide lactique, en provoquant l'apparition de divers désagréments. Le vin devient filant (à cause de la présence du ferment de la graisse), ou totalement désacidifié (dû au ferment de la tourne) ou encore amer (à cause du ferment de l'amertume).

Tableau 1: Résultats des contrôles microbiologiques des différents types de vins embouteillés

\begin{tabular}{|l|c|c|c|c|c|}
\hline Paramètre & Vin rouge & Vin blanc & $\begin{array}{l}\text { Vin blanc } \\
\text { moelleux }\end{array}$ & Vin rosé & Vin gris \\
\hline Mycoderma vini & $(-)$ & $(-)$ & $(-)$ & $(-)$ & $(-)$ \\
\hline Mycoderma aceti & $(-)$ & $(-)$ & $(-)$ & $(-)$ & $(-)$ \\
\hline Ferment de la tourne & $(-)$ & $(-)$ & $(-)$ & $(-)$ & $(-)$ \\
\hline Ferment de l'amertume & $(-)$ & $(-)$ & $(-)$ & $(-)$ & $(-)$ \\
\hline Ferment de la graisse & $(-)$ & $(-)$ & $(-)$ & $(-)$ & $(-)$ \\
\hline
\end{tabular}
$(+)$ : Présence $(-)$ : Absence

\section{CONCLUSION}

La composition des grains de raisin dépend fortement de la variété du cépage, du terrain sur lequel se trouve la vigne ainsi que des conditions climatiques de l'année. Le goût du vin est donc influencé par la composition du raisin, la façon de vinifier, le genre de ferments utilisés, la durée et la température du vieillissement. Il en résulte que chaque vin est différent et a un bouquet qui lui est propre. L'évaluation de la qualité des vins sont plus sensibles aux différences en matière de choix technologiques qu'aux spécificités du terroir. La viticulture a subit éventuellement 
l'effet du changement climatique. La pluie d'hiver est favorable à la maturation du raisin tandis que la forte pluie avant le vendage et au moment de la maturation dégradent sa qualité. La teneur en sucres contenus dans les moûts devient assez faible. De ce fait, on a souvent recours à la chaptalisation dans le procédé, notamment pour les vins rouges. L'acidité du vin est plus importante, d'un point de vue analytique et organoleptique. Nos vins possèdent une acidité légèrement supérieure par rapport aux vins de qualité connue. Celle-ci influence la texture, les caractéristiques organoleptiques ainsi que la stabilité microbiologique des vins produits. La faible teneur en anhydride sulfureux peut entrainer l'altération de la qualité des vins, surtout lors du stockage à froid. A cet effet, il

\section{RÉFÉRENCES BIBLIOGRAPHIQUES}

Akin $\mathrm{H}, 2008$. Evolution du pH pendant la fermentation alcoolique de moûts de raisins : modélisation et interprétation métabolique. Thèse de doctorat. Institut national polytechnique de Toulouse. ED : Mécanique Energétique Génie Civil et Procédés. $136 \mathrm{pp}$.

Bagard $M, 2010$. Règlement Régional du Concours Général Agricole 2010 concernant les vins produits dans les départements de la Corse du Sud et de la Haute Corse. Direction départementale de l'agriculture et de la foret de la haute Corse. 9 pp.

Carette J, 2005. De la grappe au verre. éd Publibook. Vol 1. Paris. 554 pp.

Cole-Parmer, 2017. Mesurer le pH lors de la vinification. Technical Resource Library. . Consulté le 21 janvier 2018. Antananarivo. https://www .coleparmer.ca/tech-article/measuring-ph-inwine-making?tlg=fr-FR

Delanoë D, Maillard C, Maisondieu D, 2007. Le vin : De l'analyse à l'élaboration. ISBN 2-7430-0497-5, Tec \& Doc Lavoisier, Londres-Paris-New-York. $206 \mathrm{pp}$.

Escudier JL et al, 2016. New vineyard fields: Grape Juice. Selection of grapevine species, juice making, stabilization. 39th World Congress of Vine and Wine. BIO Web of Conferences 7. $11 \mathrm{pp}$.

Garrier G, 1998. Histoire sociale et Culturelle du Vin. Collection In Extenso, édition Larousse-Bordas. $770 \mathrm{pp}$.

Gerbaud V, 1996. Détermination de l'état de sursaturation et effet des polysaccharides sur la cristallisation du bitartrate de potassium dans est important de réguler la teneur en anhydride sulfureux pour stabiliser les vins produits embouteillés afin de garantir la qualité de produits par rapport aux vins importés. Le secteur vinicole à Madagascar rencontre certaines difficultés. On reproche aux domaines vinicoles malgaches d'être pauvres en technologie qu'en diversité. L'innovation technologique offre la possibilité aux différents acteurs de la filière vitivinicole de cette région de mettre en place des procédures de contrôle qualité à tous les niveaux. La création d'un laboratoire national agréé par l'Office international du vin et la mise en place d'une législation spécifique sont donc attendues pour recadrer le secteur.

les vins. Thèse de doctorat, Institut National Polytechnique de Toulouse. $236 \mathrm{pp}$.

Harry WP, 1996. Science, Vine and Wine in modern France. Press syndicate of the Cambridge University. ISBN 0-521-49745-0. 362 pp.

Hudelson J, 2011. Wine faults : causes, effects, cures. ISBN 978-1-934259-63-4, Edition Board and Bench Publishing, USA. 78 pp.

IGP, 2011. Cahier des charges de l'Indication Géographique Protégée " côtes du lot ". Consulté le 15 Octobre 2017. Antananarivo. https://www.inao.gouv.fr/fichier/PNO2CDCIGPC otesduLot.pdf

ITV France, 2002. La maîtrise du sulfitage des moûts et des vins. Les cahiers itinéraires d'Itv France, $\mathrm{n}^{\circ} 3,20 \mathrm{pp}$.

Navarre C, 1998. L'œnologie. 4e éd, Tec \& Doc, Paris. $280 \mathrm{pp}$.

Ndrasana RT, 1999. Contribution à l'étude de quelques échantillons de vins blancs malagasy, recherche de falsification. Mémoire de DEA en chimie physique, Faculté des Sciences - Université d'Antananarivo. $90 \mathrm{pp}$.

OIV, 2006. Résolution œnologie. Edition OIV, Paris. 15 $\mathrm{pp}$.

OIV, 2015. Recueil international des méthodes d'analyse des vins et des moûts, vol 1. Edition OIV Paris. $523 \mathrm{pp}$.

Rajaona HP, 1999. Contribution à l'étude physicochimique de vins rouges commerciales à Madagascar. Mémoire de DEA en Chimie Physique, Faculté des Sciences - Université d'Antananarivo. 92 pp. 
Razafindrabe JT, 2010. Contribution à l'étude des vins de Madagascar par analyse directe des mélanges complexes des extraits utilisant la RMN du carbone-13 assistée par ordinateur. Thèse de doctorat en Chimie Physique, Faculté des Sciences - Université d'Antananarivo. 146 pp.

Schopfer JF, 1998. Laboratoire d'œnologie. 1ère Partie. Edition Changins. $50 \mathrm{pp}$.

Tusseau D et Feneuil A, 1992. Stabilité tartrique des vins. Comparaison de quelques appareils de mesure. Le Vigneron Champenois, $n^{\circ} 4,10 \mathrm{pp}$.

UE, 2009. Catégories de produits de la vigne, les pratiques œnologiques et les restrictions qui s'y appliquent. RĖGLEMENT (CE) N o 606/2009. Journal officiel de l'Union Européen. Consultéle 21 janvier 2018. Antananarivo. http://eurlex.europa.eu/legal-content/FR/ALL/?uri=CELEX :32009R0606

Vin Social Club, 2014. Vins Bio. Consulté le 28 janvier 2018. Antananarivo. http://www.vinsocialclub .fr/magazine- vin/dossier-du-vin/vins-bio 